\title{
Sustained Manual Loading of the Fascial System Can Evoke Tonic Reactions: Preliminary Results
}

\author{
Luiz Fernando Bertolucci, BSc, MD, ${ }^{1}$ Elisa Harumi Kozasa, $\mathrm{PhD}^{2}$ \\ ${ }^{1}$ Associação Brasileira de Rolfing*, and ${ }^{2}$ Núcleo de Estudos em Saúde Coletiva e da Família-Universidade Nove de \\ Julho, Depto. de Psicobiologia-Universidade Federal de São Paulo, São Paulo, Brazil
}

Introduction: The physiologic mechanisms influenced or activated by manual therapies are poorly understood, and consequently, most approaches lack objectivity both in diagnostics and treatment. However, a myofascial release technique called Muscle Repositioning (MR) appears to evoke specific and detectable mechanical and neural reactions. A previously published study set forth the recorded increase in tonic activity of the cervical erectors during MR maneuvers in the occipital region ${ }^{(1)}$. This study investigated possible increases in tonic muscular activity in the cervical and lumbar erectors during an MR maneuver in the thoracic region.

Methods: In 14 healthy adults with a mean age of 34.21 years [standard deviation (SD): 10.24 years] the right cervical and lumbar erectors were monitored by surface electromyography [EMG (Nexus 10: Mind Media BV, Roermond-Herten, Netherlands)]. From a side-lying position, each subject received two MR maneuvers administered to the right side of the thorax by an experienced practitioner (Fig. 1.). The maneuvers delivered a particular set of forces, which characteristically produce a visually evident integration of body segments, causing them to appear unified as a single block when manually vibrated (video links available at http://musclerepositioning.blogspot.com/). Each maneuver continued until the practitioner felt a tissue release (a tactually perceptible sliding of internal soft parts relative to one another). The durations of the maneuvers were registered.

Results: The first maneuver lasted 14.75 minutes (SD: 5.22 minutes), and the second, 7.63 minutes (SD: 3.35 minutes). In 13 subjects, increased cervical erector activity was observed: in 11 , during both maneuvers, and in 2, only during the second maneuver. Such activity was more pronounced and ensued more quickly during the second maneuver (Fig. 2).

This article is the expanded abstract of a paper presented at the Second International Fascia Research Congress; October 27 - 30, 2009; Amsterdam, Netherlands.
Seven subjects also showed increased lumbar activity, which in 6 was synchronous with the increased

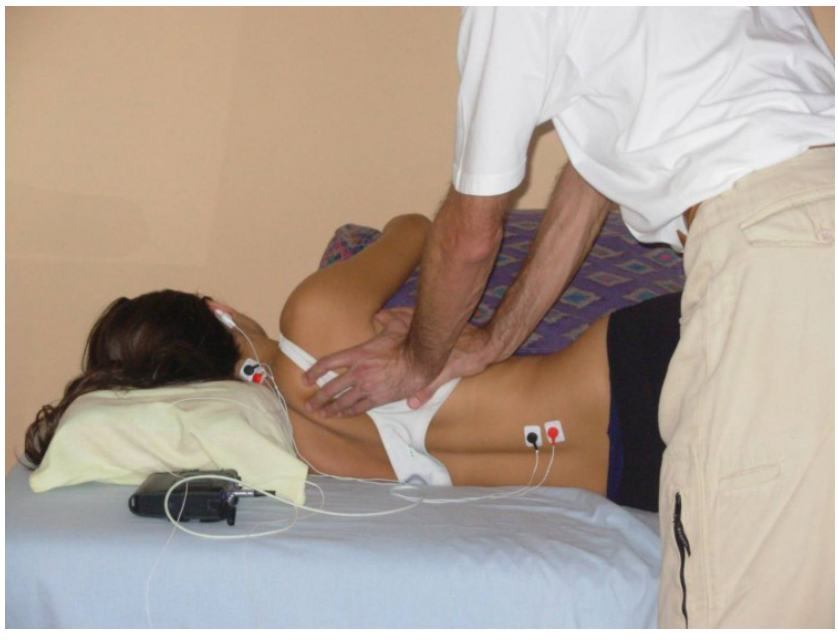

FIG. 1. Muscle Repositioning thoracic maneuver: The practitioner delivers a particular set of forces, which characteristically produce the integration of body segments, causing them to appear unified as a single block when manually oscillated. Electromyography monitors cervical and lumbar right erectors.

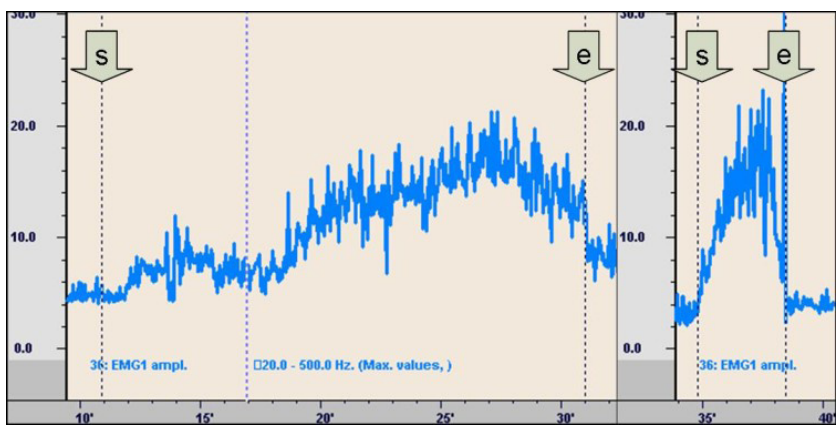

FIG. 2. Cervical electromyographic (EMG) readings of two thoracic Muscle Repositioning (MR) maneuvers: The signal becomes progressively higher, peaks at or near the conclusion of the maneuver and drops thereafter. The EMG activity was more pronounced and ensued more quickly during the second maneuver. Signal is in microvolts; time is in minutes. $s=$ start of maneuver; $e=$ end of maneuver.

* Rolfing is a service mark of the Rolf Institute of Structural Integration, Boulder, CO, USA 
cervical activity (Fig. 3). Characteristically, the muscular reactions became progressively stronger, as did the degree of apparent segmental integration. The muscular reactions and the segmental integration often both peaked at or near the conclusion of the maneuver - that is, upon tissue release-and dropped thereafter.

Discussion: Manually Induced Tonic Reactions: Our observations support the possibility that specific and sustained manual input can evoke tonic reflexes, and the synchronicity between lumbar and cervical reactions suggests that such tonic reflexes are systemic. The greater responsiveness of the cervical region when compared with the lumbar segment may be related to a richer proprioceptive innervation of the former. The smaller latency and larger EMG responses in the second maneuver suggest that the

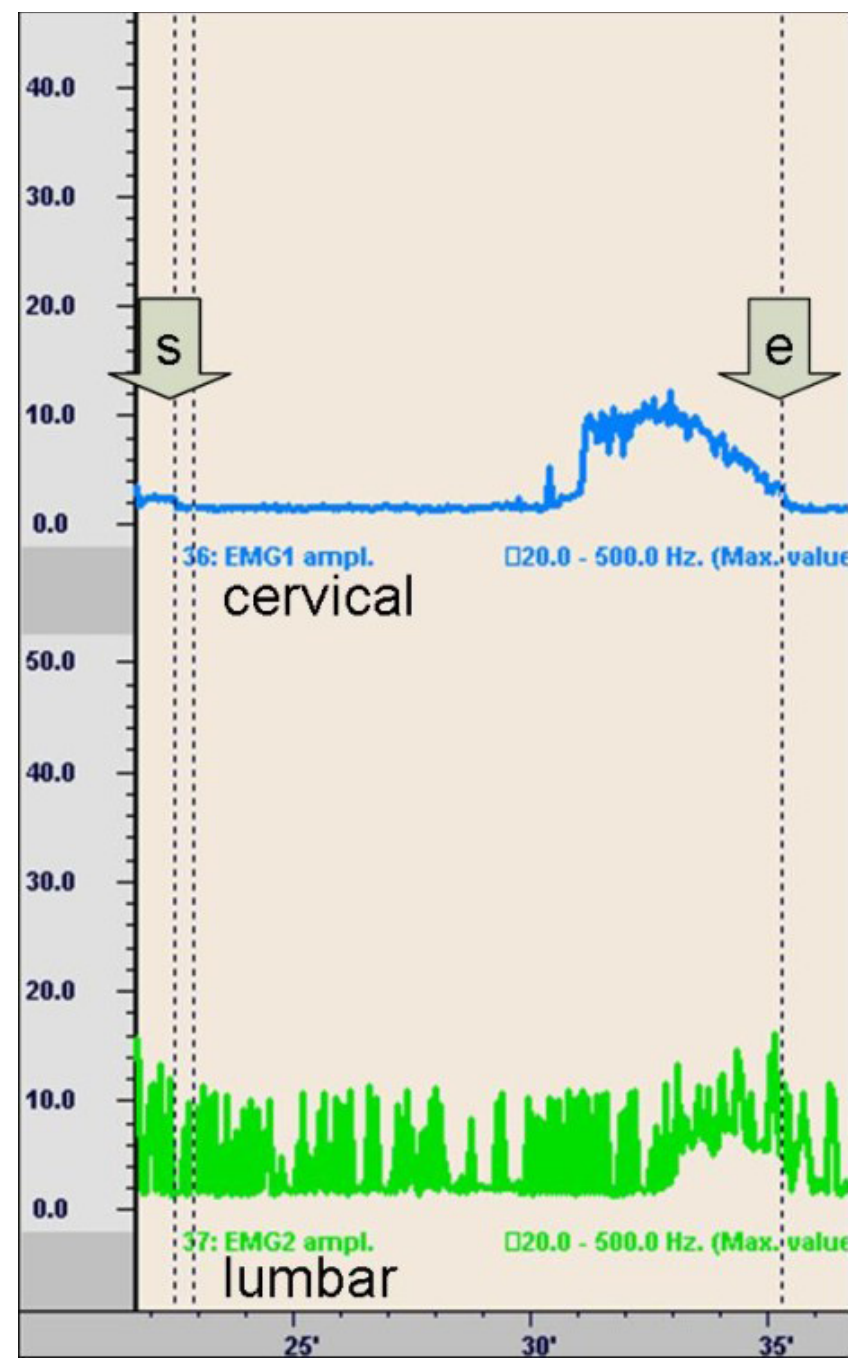

FIG. 3. Cervical and lumbar electromyographic (EMG) readings of one Muscle Repositioning (MR) thoracic maneuver: Synchronic cervical and lumbar activity illustrate the systemic feature of the involuntary muscle reactions. Signal is in microvolts; time is in minutes. $s=$ start of maneuver; $e=$ end of maneuver. first maneuver should have enhanced the sensitivity of the involved circuits to a lower threshold. Manual forces applied are believed to tense the fascial system, stimulating a progressive, larger chain of reflexes, up to the point of tissue release. The physiological significance of these recorded phenomena remains unknown, but taken together with observations from MR clinical practice, these phenomena are reminiscent of pandiculation.

Pandiculation-like Reflexes? Pandiculation, which includes yawning, is the instinctual soft-tissue stretching almost ubiquitous in animals (Fig. 4) that is believed to underlie neuromusculoskeletal development and maintenance ${ }^{(2)}$. During pandiculation, when soft tissues are vigorously stretched (the Latin pandiculare means "stretch"), a progressive and involuntary tonic activity gradually unites body segments in a block, up to an acme. Curiously, a similar progressive involuntary tonic action, up to an acme (tissue release in this case) is observed during MR maneuvers, suggesting a connection between these phenomena. Moreover, the subjective experiences of the recipients were reported to be similar to pandiculation.

A REFLEX-BASED MYOFASCIAL RELEASE TECHNIQUE? Taken together, these facts suggest that MR may be considered a blend of myofascial release with a sort of "assisted pandiculation." Therefore, the forces available for tissue release may be the sum of the therapist's manual input with the client's self-generated forces of the tonic pandiculation-like reflexes. Such features could account for a high level of clinical efficacy in the treatment of musculoskeletal disorders. If so, as the outcomes of MR clinical practice also suggest, MR would appear to have therapeutic utility. Moreover, the ability to monitor physiologic responses in real time may bring objectivity to MR and possibly also to other manual techniques.

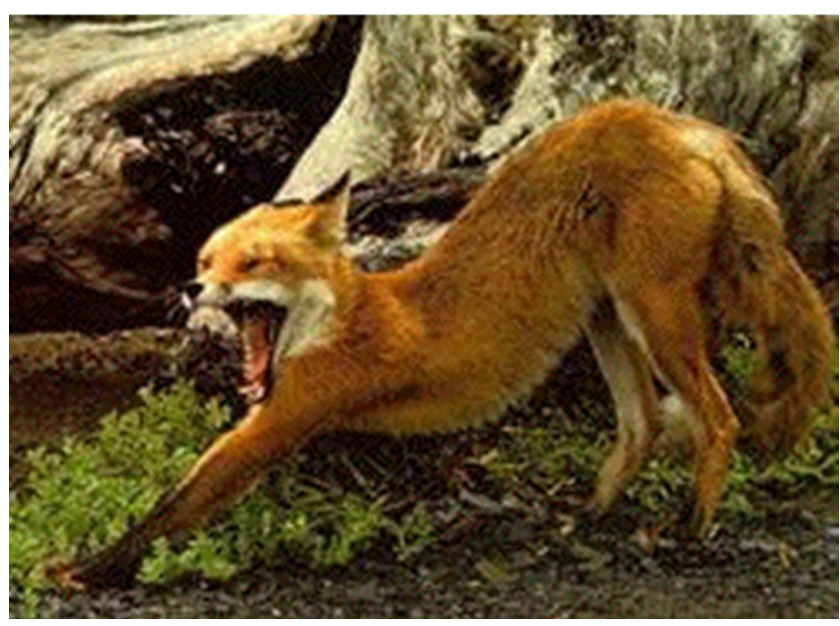

FIG. 4. A pandiculating fox. (From http://yawning.info, reproduced with permission). 
Martial Arts, Yoga and Pandiculation: Further Reflections About Health Maintenance: During MR practice, manually uniting bodily segments in a block produces a characteristic and palpable firmness of the client's body. Both aspects (linking of body segments and firmness) resemble features cultivated in some Eastern disciplines, such as styles of qi gong and yoga. Furthermore, as with pandiculation, they are believed to stimulate physiological self-maintenance mechanisms, besides advocating specific attentional states that evoke spontaneous motor expressions. Taken together, these facts suggest connections between Eastern disciplines, MR, and pandiculation. The latter, still poorly understood in its physiological significance, may be an overlooked mechanism in health maintenance.

KEYWORDS: Manual loading, fascia, fascial system, tonic reactions

\section{ACKNOWLEDMENT}

The authors thank Luis Paulo Cotrim Amorim, Heidi Massa, Yeda Bocaletto, Angela Lobo, and Soraia Pacchioni.

\section{CONFLICT OF INTEREST NOTIFICATION}

The author declares that there are no conflicts of interest.

\section{COPYRIGHT}

Published under the CreativeCommons Attribution-NonCommercial-NoDerivs 3.0 License.

\section{REFERENCES}

1. Bertolucci LF. Muscle Repositioning: a new verifiable approach to neuro-myofascial release? J Bodyw Mov Ther 2008; 12(3): 213-224.

2. Walusinski O. Yawning: unsuspected avenue for a better understanding of arousal and interoception. Med Hypotheses 2006; 67(1): 6-14.

Corresponding author: Luiz Fernando Bertolucci, Avenida Dr Arnaldo, 1644, Sumaré 01255-000 São Paulo, SP, Brazil.

E-mail: bertolucci.lf@gmail.com 